Bongartz

Markenführung im Internet 
Schriftenreihe

\section{Unternehmensführung und Marketing}

Herausgeber:

Prof. Dr. Dr. h. c. mult. Heribert Meffert, Münster/Westf.

Prof. Dr. Hartwig Steffenhagen, Aachen

Prof. Dr. Hermann Freter, Siegen

\section{Band 42}

Eine Aufstellung der in dieser Schriftenreihe erschienenen Bände finden Sie am Ende des Buches. 
Michael Bongartz

\section{Markenführung \\ im Internet}

Verhaltenstypen - Einflussfaktoren Erfolgswirkungen

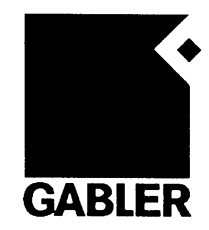


Bibliografische Information Der Deutschen Bibliothek

Die Deutsche Bibliothek verzeichnet diese Publikation in der Deutschen Nationalbibliografie; detaillierte bibliografische Daten sind im Internet über <http://dnb.ddb.de> abrufbar.

D6 (2002)

\section{Auflage Oktober 2002}

Alle Rechte vorbehalten

(C) Springer Fachmedien Wiesbaden 2002

Ursprünglich erschienen bei Betriebswirtschaftlicher Verlag Dr. Th. Gabler GmbH, Wiesbaden 2002

Lektorat: Barbara Roscher / Jutta Hinrichsen

www.gabler.de

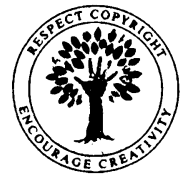

Das Werk einschließlich aller seiner Teile ist urheberrechtlich geschützt. Jede Verwertung außerhalb der engen Grenzen des Urheberrechtsgesetzes ist ohne Zustimmung des Verlages unzulässig und strafbar. Das gilt insbesondere für Vervielfältigungen, Übersetzungen, Mikroverfilmungen und die Einspeicherung und Verarbeitung in elektronischen Systemen.

Die Wiedergabe von Gebrauchsnamen, Handelsnamen, Warenbezeichnungen usw. in diesem Werk berechtigt auch ohne besondere Kennzeichnung nicht zu der Annahme, dass solche Namen im Sinne der Warenzeichen- und Markenschutz-Gesetzgebung als frei zu betrachten wären und daher von jedermann benutzt werden dürften.

Gedruckt auf säurefreiem und chlorfrei gebleichtem Papier

ISBN 978-3-409-12331-0

ISBN 978-3-663-10729-3 (eBook)

DOI 10.1007/978-3-663-10729-3 
Meinen Eltern

und

Ramona 


\section{Geleitwort}

Die zunehmende Verbreitung und Nutzung des Internet als neuartigem Informations-, Kommunikations-, Distributions- und Transaktionskanal stellen Unternehmen vor zahlreiche neue Herausforderungen. In jüngster Zeit werden in diesem Zusammenhang die Einsatzmöglichkeiten des Internet im Rahmen der Markenführung von Unternehmen besonders intensiv diskutiert. Dabei herrscht sowohl in der Marketingwissenschaft als auch in der Unternehmenspraxis weitgehend Einigkeit darüber, dass das Internet in Zukunft einen unverzichtbaren Bestandteil moderner Markenführungskonzepte bilden wird. Der wachsenden Bedeutung dieses interaktiven und innovativen Mediums im Rahmen markenpolitischer Aktivitäten steht jedoch eine bislang eher rudimentäre Behandlung durch die Marketingwissenschaft gegenüber. Gleichzeitig sieht sich offenbar eine Vielzahl von Unternehmen mit Problemen hinsichtlich der Führung ihrer Marken im Internet konfrontiert, die in Form unkoordinierter Markenauftritte im Internet und einer mangelnden Ausschöpfung der medieninhärenten Potenziale zur Markenprofilierung zu Tage treten.

Vor diesem Hintergrund hat sich der Verfasser das Ziel gesetzt, einen umfassenden Ansatz zur Erfassung und Beschreibung des internetbezogenen Markenführungsverhaltens von Unternehmen zu entwickeln, Einflussfaktoren des Markenführungsverhaltens im Internet zu identifizieren und zudem die Erfolgswirkungen markenpolitischer Internetaktivitäten zu analysieren. Dieser Zielsetzung folgend bilden die Entwicklung und empirische Überprüfung eines Modells der internetbezogenen Markenführung, welches dazu dient, das Markenführungsverhalten von Unternehmen im Internet ganzheitlich abzubilden, den Ausgangspunkt der vorliegenden Untersuchung. Auf der Grundlage dieses Modells weist der Autor fünf Markenführungstypen nach, die sich durch divergierende Verhaltensmuster hinsichtlich ihrer internetbezogenen Markenführung auszeichnen. Als zentrale Einflussgrößen der unterschiedlichen Verhaltensweisen stellen sich die übergeordneten markenstrategischen Ziel- und Positionierungsvorgaben sowie die jeweilige Nutzer- und Konkurrenzsituation der betrachteten Unternehmen heraus. Die Analyse der Erfolgswirkungen des internetbezogenen Markenführungsverhaltens führt zu der Erkenntnis, dass die medienübergreifende Vernetzung der Internetaktivitäten mit anderen markenpolitischen Maßnahmen zum entscheidenden Stellhebel bzw. zur Voraussetzung eines erfolgreichen Internetengagements avanciert. Aufbauend auf diesen Ergebnissen formuliert der Autor Implikationen für eine zielsetzungsgerechte Ausgestaltung der Markenführung im Internet durch Unternehmen 
und zeigt Ansatzpunkte für weiterführende Forschungsarbeiten auf dem Gebiet der Markenführung im Internet auf.

Insgesamt gesehen werden durch die vorliegende Untersuchung mit ihrer empirisch fundierten Analyse beachtliche Erkenntnisfortschritte über das internetbezogene Markenführungsverhalten von Unternehmen generiert. Der Verfasser setzt sich in konstruktiv-kritischer Weise mit existierenden, vorwiegend partiellen Forschungsbemühungen auf diesem Gebiet auseinander und entwickelt ein anspruchsvolles, eigenständiges Modell der Markenführung im Internet. Diese Vorgehensweise gibt der Arbeit eine fundierte Basis für die empirische Analyse der Einflussgrößen und Erfolgswirkungen der internetbezogenen Markenführung. Angesichts des bisherigen Forschungsstandes kommt der Arbeit der Charakter einer Basisuntersuchung zu, die sowohl eine Bereicherung der wissenschaftlichen Diskussion darstellt als auch für die Gestaltung markenpolitischer Internetaktivitäten in der Praxis von Nutzen ist.

Die empirische Analyse der vorliegenden Arbeit erfolgte auf der Basis des Datenmaterials eines vom Institut für Marketing der Westfälischen Wilhelms-Universität Münster in Zusammenarbeit mit der Gesellschaft für Konsum- und Absatzforschung (GfK Nürnberg) durchgeführten Forschungsprojektes. Ohne die umfassende Förderung dieses Projektes durch die GfK Nürnberg wäre die Realisierung der Untersuchung in dieser Form nicht möglich gewesen. Hierfür gilt mein besonderer Dank. 


\section{Vorwort}

Die Führung von Marken hat sich in den vergangenen Jahren zu einem Schlüsselthema und Kernbereich des Marketing entwickelt. In diesem Kontext wird dem Internet - angesichts der rasanten Entwicklung der Nutzerzahlen und der Nutzungsintensität - gerade in der Unternehmenspraxis besondere Aufmerksamkeit als innovativem und interaktivem Profilierungsraum für Marken gewidmet. So existiert kaum noch ein Unternehmen, welches nicht einen Internetauftritt für die von ihm geführten Marken eingerichtet hat. Gleichwohl offenbaren sich bei der Nutzung dieses neuen Mediums zahlreiche Schwierigkeiten. Vom „Markenmord im Internet" ist die Rede: die Potenziale des Mediums werden demnach häufig unzureichend ausgeschöpft und eine außen- und innengerichtete Verzahnung mit anderen markenpolitischen Maßnahmen findet oftmals nur sporadisch statt. Den in der Praxis zu beobachtenden vielfältigen und äußerst heterogenen markenbezogenen Internetaktivitäten stehen aus wissenschaftlicher Perspektive zudem bislang eher partielle und in den Anfängen befindliche Forschungsbemühungen gegenüber.

Die vorliegende Arbeit knüpft an den angesprochenen wissenschaftlichen und praktischen Defiziten auf dem Gebiet der Markenführung im Internet an. Auf theoretisch-konzeptioneller sowie empirischer Basis wird unter Bezugnahme auf das Konzept der identitätsorientierten Markenführung zunächst angestrebt, einen grundlegenden Ansatz zur Erfassung und Charakterisierung des internetbezogenen Markenführungsverhaltens von Unternehmen zu entwickeln. Darüber hinaus wird das Ziel verfolgt, eine systematische Analyse der Einflussfaktoren und Erfolgswirkungen alternativer Ausprägungen des Markenführungsverhaltens im Internet vorzunehmen.

Diesen Ansprüchen wird in mehreren Arbeitsschritten Rechnung getragen. Ausgehend von den als konstitutiv erachteten Handlungsfeldern des internetbezogenen Markenführungsverhaltens - der außengerichteten Markengestaltung und Markenintegration sowie der innengerichteten Markenkoordination - werden neun zentrale Verhaltensdimensionen abgeleitet und in einem ganzheitlichen Modellansatz der Markenführung im Internet zusammengeführt. Dieser empirisch verifizierte Modellansatz bildet die Ausgangsbasis für die weitere Untersuchung. Nach der modellbasierten Identifikation von fünf internetbezogenen Markenführungstypen, welche sich durch divergierende Verhaltensmuster auszeichnen, werden potenzielle interne und externe Einflussfaktorenkategorien hinsichtlich ihrer 
Erklärungsbeiträge für die Herausbildung dieser Typen analysiert. Den Abschluss bildet die Untersuchung von Erfolgswirkungen des internetbezogenen Markenführungsverhaltens sowohl auf disaggregierter Ebene der einzelnen Verhaltensdimensionen als auch auf aggregierter Ebene der aufgedeckten Markenführungstypen. Die auf diese Weise gewonnenen Erkenntnisse dienen als Ausgangspunkt für die Formulierung von Implikationen für die unternehmerische Praxis. Darüber hinaus werden zahlreiche Ansatzpunkte für weiterführende Forschungsarbeiten auf dem Gebiet der internetbezogenen Markenführung aufgezeigt.

Das Entstehen dieser Arbeit war nur mit der Unterstützung zahlreicher Personen und Institutionen möglich. Mein besonderer Dank gilt zunächst meinem akademischen Lehrer, Herrn Prof. Dr. Dr. h.c. mult. Heribert Meffert, der meine fachliche und persönliche Entwicklung als wissenschaftlicher Mitarbeiter am Institut für Marketing umfassend förderte. Er gab nicht nur frühzeitig entscheidende Impulse bei der Auswahl und Konkretisierung der Themenstellung, sondern unterstützte die Erstellung der Arbeit auch in allen weiteren Phasen ihrer Entstehung. Herrn Prof. Dr. Stefan Klein danke ich herzlich für die Übernahme des Zweitgutachtens.

Die empirischen Analysen der Arbeit basieren auf einer umfassenden Befragung markenführender Unternehmen in Deutschland. Die Durchführung dieser Anbieterbefragung wäre ohne die Unterstützung der Gesellschaft für Konsum- und $A b-$ satzforschung (GfK Nürnberg) in der vorliegenden Stichprobengröße und in der erreichten Qualität nicht möglich gewesen. Daher möchte ich mich an dieser Stelle nachdrücklich bei Herrn Dr. Raimund Wildner und seinen Mitarbeitern für die kooperative Zusammenarbeit und die professionelle Erhebung der Daten bedanken.

Dank gebührt nicht zuletzt auch allen aktuellen und ehemaligen Mitarbeiterinnen und Mitarbeitern am Institut für Marketing der Westfälischen Wilhelms-Universität Münster. Sie haben mich während der Abfassung meiner Dissertationsschrift in vorbildlicher Weise unterstützt und mich von anderweitigen Verpflichtungen weitgehend entlastet. Hervorzuheben ist dabei mein Kollege und Freund, Herr Dipl.$\mathrm{Kfm}$. Bastian Grunberg. Er war trotz eigener hoher Arbeitsbelastung stets ein kompetenter, engagierter und aufmunternder Diskussionspartner, der durch seine Anregungen maßgeblich zur Qualität dieser Arbeit beigetragen hat. Ferner gilt mein Dank auch Herrn Dr. Christian Böing, der sich neben seinen vielfältigen beruflichen Aufgaben die Zeit nahm, mich in kürzester Zeit in die Anwendung der erforderlichen statistischen Software einzuführen. Darüber hinaus bedanke ich mich auch bei meinen Freundinnen und Freunden außerhalb des Instituts, die mir 
während der gesamten Promotionszeit ein Höchstmaß an Verständnis entgegengebracht haben.

Ganz besonderer Dank gilt meiner Familie, insbesondere meinen Eltern, die mir in allen Phasen meiner Ausbildung stets den notwendigen Rückhalt gegeben und mir in vielerlei Hinsicht mit „Rat und Tat“ zur Seite gestanden haben. Dabei ließen sie mir nicht nur immer die Freiheit eigener Entscheidungen, sondern haben durch ihre liebevolle Unterstützung und Förderung meine berufliche Laufbahn und damit meine Promotion erst ermöglicht.

Der letzte Absatz gilt Dir, liebe Ramona. Du hast nicht nur die Auswirkungen der Dissertation auf unser Privatleben mit großer Gelassenheit und Nachsicht ertragen, sondern mich auch - wie selbstverständlich - von allen sonstigen Arbeiten entlastet. Während der zahlreichen „Höhen und Tiefen“ haben mir Deine Ausgeglichenheit und Dein Zuspruch geholfen, den Blick für das Wesentliche niemals aus den Augen zu verlieren. 


\section{Inhaltsverzeichnis}

Abbildungsverzeichnis XVII

Tabellenverzeichnis

XIX

Abkürzungsverzeichnis. $X X I$

A. Internet als Herausforderung an die Markenführung ......................... 1

1. Bedeutung des Internet für die Führung von Marken............................. 1

2. Internet als integrativer Bestandteil der Markenführung......................... 9

2.1 Identitätsorientierte Markenführung als Ausgangspunkt ............... 9

2.2 Internetbezogene Markenführung als Gegenstand der Untersuchung....

2.3 Bestandsaufnahme von Forschungsarbeiten mit Bezug zur internetbezogenen Markenführung

3. Zielsetzung und Gang der Untersuchung ........................................... 28

4. Design und Methodik der empirischen Analyse .................................. 33

4.1 Datenerhebung und Datenbasis ........................................ 33

4.2 Methoden und Beurteilungskriterien der statistischen Auswertung

B. Bildung einer internetbezogenen Markenführungstypologie auf Basis von Verhaltensdimensionen.

1. Anforderungen an eine Typologie der internetbezogenen Markenführung und Analyse bestehender Beschreibungs- und Typologisierungsansätze

2. Ableitung von Verhaltensdimensionen der internetbezogenen Markenführung als Grundlage einer empirischen Führungstypologie...... 51

2.1 Dimensionen der internetbezogenen Markengestaltung .............. 53

2.2 Dimensionen der internetbezogenen Markenintegration.............. 72

2.3 Dimensionen der internetbezogenen Markenkoordination ............ 77 
2.4 Integration der Verhaltensdimensionen in ein Modell der internetbezogenen Markenführung

3. Empirische Überprüfung des Modells der internetbezogenen Markenführung und Identifikation von Markenführungstypen

3.1 Konfirmatorische Faktorenanalyse für das Modell der internetbezogenen Markenführung

3.2 Bildung und Analyse internetbezogener Markenführungstypen .... 93

3.2.1 Ermittlung der Anzahl unterschiedlicher Markenführungstypen. 93

3.2.2 Darstellung der identifizierten Markenführungstypen 98

3.2.2.1 Funktional-informelles Markenführungsverhalten

3.2.2.2 Universell-hochintegriertes Markenführungsverhalten

3.2.2.3 Bewusst-divergentes Markenführungsverhalten.. 100

3.2.2.4 Transaktionsdominant-strukturelles Markenführungsverhalten 101

3.2.2.5 Minimalistisch-autonomes Markenführungsverhalten

3.2.3 Diskriminanzanalytische Überprüfung der Clusterlösung und Ermittlung der zur Typendifferenzierung zentralen Verhaltensdimensionen

C. Analyse der Einflussfaktoren und Erfolgswirkungen des internetbezogenen Markenführungsverhaltens

1. Theoretische Ableitung und empirische Überprüfung von

Einflussfaktoren des internetbezogenen Markenführungsverhaltens

1.1 Systematisierung von Einflussfaktoren des internetbezogenen Markenführungsverhaltens

1.1.1 Nutzer- und Konkurrenzsituation als zentrale externe Einflussfaktoren des internetbezogenen Markenführungsverhaltens

1.1.2 Interne Einflussfaktoren des internetbezogenen Markenführungsverhaltens 
1.1.2.2 Stellenwert des Internet im Rahmen der Markenführung

1.1.2.3 Einsatz unternehmensfremder Berater 125

1.1.2.4 Unternehmensdemographie 126

1.2 Empirische Analyse der Einflussfaktoren der internetbezogenen Markenführung.

1.2.1 Analyse des Einflusses der Nutzer- und Konkurrenzsituation 130

1.2.2 Analyse der internen Einflussfaktoren 136

1.2.2.1 Einflüsse des markenstrategischen Handlungsrahmens

1.2.2.2 Einflüsse des Stellenwertes des Internet im Rahmen der Markenführung....

1.2.2.3 Einflüsse des Einsatzes unternehmensfremder Berater.

1.2.2.4 Einflüsse der Unternehmensdemographie 147

1.2.3 Integrierte Analyse der Einflussfaktoren des internetbezogenen Markenführungsverhaltens

2. Konzeptionelle Analyse und empirische Überprüfung von

Erfolgswirkungen des internetbezogenen Markenführungsverhaltens.... 153

2.1 Erfassung des Erfolges der internetbezogenen Markenführung als Ausgangspunkt der Analyse

2.2 Disaggregierte Analyse und empirische Überprüfung von Erfolgswirkungen des internetbezogenen Markenführungsverhaltens

2.2.1 Ableitung von Erfolgswirkungen auf der Ebene der Verhaltensdimensionen.

2.2.1.1 Direkte und moderierende Wirkungen außengerichteter Verhaltensdimensionen auf die Markenbekanntheit

2.2.1.2 Direkte und moderierende Wirkungen außengerichteter Verhaltensdimensionen auf das Markenimage. 
2.2.1.3 Direkte und moderierende Wirkungen außengerichteter Verhaltensdimensionen auf die Markenbindung

2.2.1.4 Indirekte Erfolgswirkungen der innengerichteten Verhaltensdimensionen

2.2.2 Empirische Überprüfung der Erfolgswirkungen des internetbezogenen Markenführungsverhaltens

2.2.2.1 Direkte und moderierende Wirkungen auf die Markenbekanntheit

2.2.2.2 Direkte und moderierende Wirkungen auf das Markenimage

2.2.2.3 Direkte und moderierende Wirkungen auf die Markenbindung.

2.2.2.4 Indirekte Erfolgswirkungen der innengerichteten Verhaltensdimensionen

\subsubsection{Zusammenfassende Würdigung der disaggregierten}

Erfolgsanalyse

2.3 Aggregierte Analyse und empirische Überprüfung der

Erfolgswirkungen des internetbezogenen

Markenführungsverhaltens auf Typenebene

D. Zusammenfassung und Implikationen der Untersuchung................. 203

1. Zusammenfassende Darstellung der Untersuchungsergebnisse........... 203

2. Implikationen für die Unternehmenspraxis ........................................ 211

3. Implikationen für weiterführende Forschungsarbeiten ....................... 213

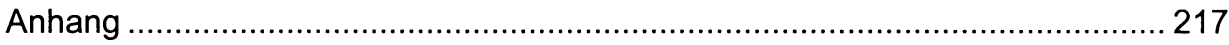

Anhang I .................................................................................. 219

Anhang II ........................................................................... 231

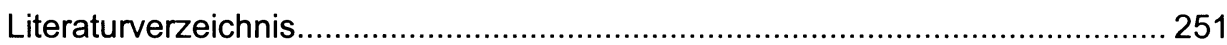




\section{Abbildungsverzeichnis}

Abbildung 1: Handlungsfelder der internetbezogenen Markenführung ............ 18

Abbildung 2: Verflechtungen zwischen klassischem Marketing-Mix und Internetaktivitäten

Abbildung 3: Zusammenfassende Darstellung der Einflussgrößen und Handlungsfelder der internetbezogenen Markenführung ............ 24

Abbildung 4: Bezugsrahmen der Arbeit.................................................... 32

Abbildung 5: Zusammensetzung der Stichprobe der Unternehmensbefragung....

Abbildung 6: Prüfschema zur Beurteilung der Güte von Strukturgleichungsmodellen

Abbildung 7: Modell der internetbezogenen Markenführung ........................... 85

Abbildung 8: Konfirmatorische Faktorenanalyse zweiter Ordnung für das Modell der internetbezogenen Markenführung ... 89

Abbildung 9: Fusionierungsprozess des Ward-Verfahrens ........................... 95

Abbildung 10: Ausprägungen der clusterbildenden Merkmale der ermittelten Markenführungstypen.

Abbildung 11: Einfluss der Nutzer- und Konkurrenzsituation auf das internetbezogene Markenführungsverhalten

Abbildung 12: Clusterbildende Merkmale der ermittelten externen Situationstypen

Abbildung 13: Konfirmatorische Faktorenanalyse für den markenstrategischen Handlungsrahmen

Abbildung 14: Einfluss des markenstrategischen Handlungsrahmens auf das internetbezogene Markenführungsverhalten

Abbildung 15: Einfluss des Stellenwertes des Internet auf das internetbezogene Markenführungsverhalten

Abbildung 16: Einflussstärke unternehmensfremder Berater differenziert nach Markenführungstypen

Abbildung 17: Umsatz und Mitarbeiterzahl differenziert nach internetbezogenen Markenführungstypen

Abbildung 18: Überblick über die Erfolgswirkungen des internetbezogenen Markenführungsverhaltens 
Abbildung 19: Kausalmodell zu direkten Wirkungen auf die Markenbekanntheit

Abbildung 20: Kausalmodell zu direkten Wirkungen auf das Markenimage ..... 185

Abbildung 21: Kausalmodell zu direkten Wirkungen auf die Markenbindung ... 188

Abbildung 22: Kausalmodell zur Überprüfung indirekter Erfolgswirkungen ...... 192

Abbildung 23: Zusammenfassender Überblick über die Erfolgswirkungen des internetbezogenen Markenführungsverhaltens.

Abbildung 24: Erfolg des internetbezogenen Markenführungsverhaltens differenziert nach Markenführungstypen 198

Abbildung 25: Synopse der disaggregierten Erfolgsanalyse 210 


\section{Tabellenverzeichnis}

Tabelle 1: Überblick über ausgewählte Arbeiten zur Führung von Marken im Internet

Tabelle 2: $\quad$ Synopse zentraler Ansätze zur Kennzeichnung des internetbezogenen Markenführungsverhaltens

Tabelle 3: Indikatoren der Verhaltensdimension „Informationsorientierung"

Tabelle 4: Indikatoren der Verhaltensdimension „Unterhaltungsorientierung“.

Tabelle 5: $\quad$ Indikatoren der Verhaltensdimension „Dialogorientierung“..... 65

Tabelle 6: Indikatoren der Verhaltensdimension „Transaktionsorientierung“. 69

Tabelle 7: Indikatoren der Verhaltensdimension „Site-Promotion“ 71

Tabelle 8: $\quad$ Indikatoren der Verhaltensdimension „Medienübergreifende Integration" 76

Tabelle 9: Indikatoren der Verhaltensdimension „Technokratischbürokratische Koordination"..... 80

Tabelle 10: Indikatoren der Verhaltensdimension „Personenorientiertdezentrale Koordination"

Tabelle 11: Indikatoren der Verhaltensdimension „Nicht-strukturelle Koordination".

Tabelle 12: Vollständigkeitsprüfung der dem Modell zugrunde gelegten

Dimensionen

Tabelle 13: Interkorrelationen der Verhaltensdimensionen 92

Tabelle 14: Klassifikationsmatrix der diskriminanzanalytischen Untersuchung der Markenführungstypen

Tabelle 15: Diskriminanzanalytische Bedeutung der Verhaltensdimensionen für die Markenführungstypen 105

Tabelle 16: Zusammenhang zwischen externen Situations- und internetbezogenen Markenführungstypen....

Tabelle 17: Zusammenhang zwischen Branchenzugehörigkeit und internetbezogenem Markenführungsverhalten 
Tabelle 18: Diskriminatorische Bedeutung der Einflussfaktoren für die

Bildung internetspezifischer Markenführungstypen 152

Tabelle 19: Moderierende Effekte hinsichtlich der Markenbekanntheit ........ 184

Tabelle 20: Moderierende Effekte hinsichtlich des Markenimages .............. 187

Tabelle 21: Moderierende Effekte hinsichtlich der Markenbindung .............. 190

Tabelle 22: $\quad$ Erfolg der internetbezogenen Markenführungstypen

differenziert nach Markenführungs- und externen

Situationstypen ............................................................. 201

Tabelle 23: Synopse der identifizierten Markenführungstypen.................... 205

Tabelle 24: $\quad$ Synopse der Einflussfaktorenanalyse für die ermittelten

Markenführungstypen. 


\section{Abkürzungsverzeichnis}

a. a. O. a. M.

AG

AGFI

AMA

AMOS

Aufl.

Bd.

bzw.

ca.

d. h.

DBW

DEV

df

$D M$

DPMA

e. V.

EDV

EQS

et al.

etc.

f.

ff.

FAQ

FAZ

FR

FTP

GFI

GfK

ggü.

GLS

$\mathrm{GmbH}$

Hrsg.

IMMF

Iwd am angegebenen Ort

am Main

Aktiengesellschaft

Adjusted Goodness of Fit Index

American Marketing Association

Analysis of Moment Structures

Auflage

Band

beziehungsweise

circa

das heißt

Die Betriebswirtschaft

Durchschnittlich erfasste Varianz

Degrees of Freedom (Freiheitsgrade)

Deutsche Mark

Deutsches Patent- und Markenamt

eingetragener Verein

Elektronische Datenverarbeitung

Equation based Structural Program

et alii, et alia, et alteri

et cetera

folgende

fortfolgende

Frequently Asked Questions

Frankfurter Allgemeine Zeitung

Faktorreliabilität

File Transfer Protocol

Goodness of Fit Index

Gesellschaft für Konsum- und Absatzforschung

gegenüber

Generalized Least Squares

Gesellschaft mit beschränkter Haftung

Herausgeber

Institut für Marketing-Management und -Forschung

Informationsdienst des Instituts der deutschen Wirtschaft Köln

$\mathrm{Jg}$.

Jahrgang 
Kap. Kapitel

LISREL Linear Structural Relations

MF

Mio.

Markenführung / Markenführungstyp

ML

Mrd.

MW

Millionen

Maximum Likelihood

Milliarde(n)

Mittelwert

n. s.

nicht signifikant

No.

Number

$\mathrm{Nr}$.

Nummer

o. V.

ohne Verfasser

RMR

Root Mean Residual

S.

Seite

sog.

sogenannte (n, r, s)

SPSS

Superior Performance Software System

TCP/IP Transmission Control Protocol / Internet Protocol

u. und

u. a. und andere / unter anderem

u. U.

unter Umständen

ULS

Unweighted Least Squares

URL

Uniform Resource Locator

$\mathrm{vgl}$.

vergleiche

Vol.

Volume

vs.

versus

WiSt Wirtschaftswissenschaftliches Studium

WISU

Das Wirtschaftsstudium

WWW

World Wide Web

z. B. zum Beispiel

ZFP Zeitschrift für Forschung und Praxis 